BBS Target Anticle Authors: Anderson \& Lebiere

Word counts: abstract: 69 ; main text: 1208; references: 138 ; total: 1415

\title{
The Newell Test Should Commit to Diagnosing Dysfunctions
}

\author{
William J. Clancey \\ Computational Sciences Division, MS 269-3 \\ NASA-Ames Research Center \\ Moffett Field, CA 94035
}

$650-604-2526$

william.j.clancey@nasa.gov

http://bill.clancey.name

\begin{abstract}
"Conceptual coordination" analysis bridges connectionism and symbolic approaches by positing a "process memory" by which categories are physically coordinated (as neural networks) in time. Focusing on dysfunctions and odd behaviors like slips reveals the function of consciousness, especially taken-forgranted constructive processes, different from conventional programming constructs. Newell strongly endorsed identifying architectural limits; the heuristic of "diagnose unusual behaviors" will provide targets of opportunity that greatly strengthens the Newell Test.
\end{abstract}

Anderson and Lebiere's (A\&L) article evaluates cognitive theories by relating them to criteria of functionality derived from Newell. Suppose that NT has all the right categories, but a significant architectural change is required for theoretical progress. I claim that "conceptual coordination" (Clancey 1999a) provides a better theory of memory, and that without committing to explaining cognitive dysfunctions, NT would not provide sufficient heuristic guidance for leading in this direction.

Conceptual coordination (CC) hypothesizes that the store, retrieve, and copy memory mechanism is not how the brain works. Instead, all neural categorizations are activated, composed, and sequenced "in place," with the assumption that sufficient (latent) physical connections exist to enable necessary links to be formed (physically constructed) at runtime (i.e., when a behavior/experience occurs). For example, if comprehending a natural language sentence requires that a noun phrase be incorporated in different ways, it is not moved or copied, but physically connected by activation of (perhaps heretofore unused) neural links. Effectively, Newell's "distal access" is accomplished by a capability to hold a categorization active and cncapsulate it (like a pointer) so it can be incorporated in different ways in a single construction. The no-copying constraint turns out to be extremely powerful for explaining a wide variety of odd behaviors, including speaking and typing slips, perceptual aspects of analogy formation, developmental "felt paths," multimodal discontinuity in dreams, and language comprehension limitations. CC 
thus specifies a cognitive architecture that bridges connectionist and symbolic concerns, and relates well to the NT criteria for which ACT-R scores weakest-development, consciousness, language, and the brain. To illustrate, I provide a diagnostic analysis of an autistic phenomenon, and then relate this back to how NT can be improved.

In $\mathrm{CC}$ analysis, a diagram notation is used to represent a behavior sequence, which corresponds in natural language to the conceptualization of a sentence. For example, according to (Baron-Cohen 1996), an autistic child can conceptualize "I stroke the cat that drinks the milk." In one form of the CC notation, a slanting line to the right represents categorizations activated sequentially in time (e.g., "I $\rightarrow$ stroke" in Figure 1). Another sequence may qualify a categorization (e.g., "the cat $\rightarrow$ drinks" qualifies "stroke"). This pattern of sequences with qualifying details forming compositions of sequences is occurs throughout $\mathrm{CC}$ analysis. The essential idea in $\mathrm{CC}$ is to understand how categories (both' perceptual categorizations and higher-order categorizations of sequences and compositions of them) are related in time to constitute conscious experience (Clancey1999a).

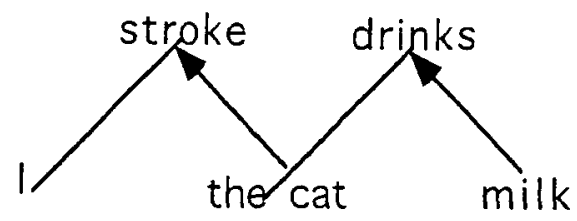

Figure 1. Unproblematic: "I stroke the cat that drinks the milk."

The challenge is to understand why an autistic child finds it problematic to conceptualize "I see the cat that sees the mouse." A traditional view is that the child lacks "social understanding." But CC analysis suggests a mechanistic limitation in the child's ability to physically sequence and compose categories. Relating to other agents requires being able to construct a 2 nd order conceptualization that relates the child's activity to the other agent's activity. Figure 2 shows the CC notation for the required construction.

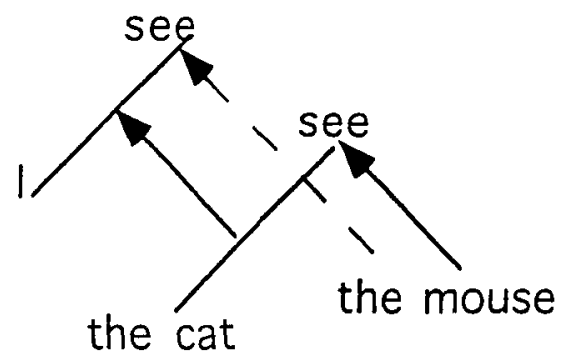

Figure 2. Problematic: "I see the cat that sees the mouse."

The statement (the conceptualization being constructed) involves a triadic relation: I see the cat, the cat sees the mouse, and I see the mouse. There is one mouse that we are both seeing. Two "see" constructions are unified by identifying a detail (the mouse) as common to both. In effect, the child must conceive of a problem space (Clancey 1999a): A common categorization of an operand (mouse) enables categorization of multiple actions as being one action (seeing), an operator. Because the two actions are by different agents, accomplishing this identification integrates perspectives of "self" (what I am 
doing now) and "other" (what that object is doing now). Indeed, the conceptualization of agent appears to be inherent in this construction.

Put another way, two sequentially occurring conceptualizations (I see the cat; the cat sees the mouse) are held active and related: "I see the cat that sees the mouse" and "I see the mouse" becomes "I see that the cat sees the mouse (i.e., the mouse that I am seeing)" (the second order relation is represented in Figure 2 by the solid arrow below "I see"). Conceiving this relation is tantamount to conceiving what joint action is. Barresi and Moore (1996) characterize this as "integrating third and first person information" (p. 148), and contrast it with (Figure 1) "embedding one third person representation in a separate first person frame" (p. 148). Related to Langacker's (1986) analysis, logical relations are not extra capabilities or meta "inference" capabilities, but generalizations of concrete accomplishments that arise through the capability to physically coordinate categories through identification, sequence, and composition in time. Mental operations are physical, subconscious processes, constrained by physical limits on how inclusion in new sequences can occur. The ability to hold two sequences active and relate them constitutes a certain kind of consciousness (e.g., not present in dreaming; Clancey 2000).

To summarize, the example requires relating sequential categorizations of seeing so they become simultaneous; it exemplifies a $2^{\text {nd }}$ order conceptualization of intentionality (my seeing is about your seeing; Clancey 1999b); and suggests that joint action requires being able to conceive the ideas we call operator and agent.

The pivotal heuristic in CC analysis is addressing "unusual" behaviors and experiences. These "targets of opportunity" appear to be deemphasized by A\&L's focus on normal behaviors "that people display on a day-to-day basis." For NT to provide heuristic guidance for discovering a theory like $\mathrm{CC}$, grading for each criteria should include diagnosing unusual phenomena that everyone experiences (e.g., slips) and dysfunctions. For example, for the criteria of Consciousness, we should direct theorization at explaining the phenomenology of dreaming, autism, compulsive-obsessive disorders, etc. For Natural Language, include comprehension difficulties (e.g., subject relatives with center-embedded noun phrases; Clancey 1999a, chapter 10). For Development, explain how "felt paths" are constructed in children's learning (chapter 5). For Knowledge Integration, explain slips (chapter 6) and "seeing as" in analogy formation (chapter 7). In this manner, learning in well-known architectures (e.g., MOPS, EPAM, SOAR) can be evaluated and the nature of problem spaces reformulated (chapter 12).

The Evolution criterion highlights the limitations of NT as stated. Rather than focusing on human evolution, this criterion should be about the evolution of cognition broadly construed, and hence should be inherently comparative across species (Clancey 1999b). Viewed this way, there is no "paucity of data," but rather a largely unexploited potential to make the study of animai cognition an integrated discipline with human problem solving. By including the heuristic "explain odd behavior" in the grading, we will naturally be guided to characterize and relate cognition in other primates, ravens, etc. This is essential for relating "instinctive" mechanisms (e.g., weaving spider webs) to brain mechanisms, development, and learned higher-order categorizations (e.g., 
conceptualization of intentionality). A\&L mention comparative considerations, but we should view this as a diagnostic problem, much as cognitive theories like $\mathrm{ACT}$ * have been used to explain students' different capabilities (Anderson et al., 1990). Furthermore, the research community should collect behaviors that have been heretofore ignored or poorly explained by computational theories, and include them in the grading criteria.

Applying the Newell Test in this way-moving from the routine behaviors already handled more or less well, to diagnostic theories that relate aberrations to architectural variations - might bring symbolic and connectionist theories together and make the study of cognition a more mature science.

\section{References}

Anderson, J., Boyle, C.F., Corbett, A.T., and Lewis, M.W. 1990. Cognitive modeling and intelligent tutoring. In W. J. Clancey and E. Soloway (eds.), Artificial Intelligence and Learning Environments, Amsterdam: Elsevier, pp. 7-50.

Baron-Cohen, S. 1996. Can children with autism integrate first and third person representations? Behavioral and Brain Sciences, 19:1, p. 123-124.

Barresi, J. and Moore, C. 1996. Intentional relations and social understanding. Behavioral and Brain Sciences, 19:1, p. 107-154.

Clancey, W. J. 1999. Conceptual Coordination: How the mind orders experience in time. Mahwah, NJ: Erlbaum.

Clancey, W. J. 1999. Studying the varieties of consciousness: Stories about zombies or the life about us? Journal of the Learning Sciences, 8(3-4):525-540.

Clancey, W. J. 2000. Conceptual coordination bridges information processing and neuropsychology. Behavioral and Brain Sciences, 23(6):919-922.

Langacker, R. W. 1986. An introduction to cognitive grammar. Cognitive Science, 10(1), $1-40$. 